\title{
Aspectos psicológicos del abordaje nutricional de los pacientes en cuidados paliativos
}

\author{
Psychological aspects of the nutritional approach of palliative care patients \\ Aspectos psicológicos da abordagem nutricional dos pacientes em cuidados paliativos
}

\author{
Laura Ramos ${ }^{1 *}$, Varinia Loureiro', Gabriela Piriz Alvarez
}

Recibido: 30 de septiembre de 2020. Aceptado para publicación: 30 de diciembre de 2020. https://doi.org/10.35454/rncm.v4n2.260

\begin{abstract}
Resumen
La desnutrición es un síndrome de alto impacto, porque es donde se materializa la enfermedad y su evolución. Esta genera repercusiones físicas y psicológicas en el paciente, así como psicológicas en la familia y en el equipo asistencial. La finalidad de este artículo es analizar la importancia de la nutrición del paciente, el abordaje integral y la repercusión en la esfera emocional no solo del paciente, sino del núcleo familiar. Luego del control de los síntomas urgentes, en orden de importancia, se maneja el resto de los síntomas físicos, los emocionales y el aspecto nutricional. La nutrición engloba lo físico y lo emocional. Para ello se considera primordial realizar un diagnóstico situacional, con base en la comunicación como herramienta fundamental. Se debe tener claro el objetivo, y acompañar en las diferentes etapas al paciente y la familia, brindando herramientas para el adecuado manejo de la evolución del paciente y su contexto.
\end{abstract}

Palabras claves: cuidados paliativos, nutrición, psicología.

\section{Summary}

Malnutrition is considered a highimpact syndrome because it is the source of the disease and its progression. Malnutrition generates physical and psychological repercussions on the patient, and psychological repercussions on the family and the healthcare team. The purpose of this article is to analyze the importance of the patient's nutrition, the comprehensive approach, and the emotional repercussion, not only on the patient, but also on his or her the family nucleus. After controlling the urgent symptoms, in order of importance, the physical and emotional symptoms and the nutritional facet are managed. Nutrition encompasses physical and emotional aspects. To achieve this, it is essential to carry out a diagnosis of the situation using communication as a basic tool, to be clear about the objective, and to accompany the patient and family in the different stages, providing tools for the adequate management of the patient's progress and his or her environment.

Keywords: Palliative care; Nutrition; Psychology.

\section{Resumo}

A desnutrição é uma síndrome de alto impacto, pois é onde se materializa a doença e sua evolução. Gera repercussões físicas e psicológicas no paciente e repercussões psicológicas na família e na equipe de saúde. A finalidade deste artigo é analisar a importância da nutrição do paciente, a abordagem integral e a repercussão na esfera emocional, não só do paciente, mas também do núcleo familiar. Depois do controle dos sintomas urgentes em ordem de importância, são gerenciados o restante dos sintomas físicos, emocionais e $\mathrm{o}$ aspecto nutricional. A nutrição abrange o físico e o emocional. Para isso, considera-se primordial a realização de um diagnóstico situacional utilizando a comunicação como ferramenta fundamental, ter clareza sobre o objetivo e acompanhar o paciente e a família nas diferentes etapas, fornecendo ferramentas para a gestão adequada da evolução do paciente e seu contexto.

Palavras-chave: cuidados paliativos, nutrição, psicologia.
Servicio de Medicina Paliativa, Hospital Maciel, Servicios de Salud del Estado (ASSE). Montevideo, Uruguay.
*Correspondencia: Laura Ramos.

medicinapaliativa.maciel@asse.com.uy 


\section{INTRODUCCIÓN}

El enfoque de atención en cuidados paliativos realiza un abordaje integral, teniendo en cuenta las dimensiones bio-psico-social-espiritual, y siguiendo un modelo asistencial integrado y continuo ${ }^{(1-5)}$. El equipo interdisciplinario de Medicina Paliativa del Hospital Maciel de Montevideo, Uruguay, equipo de referencia nacional de los servicio de salud del estado, con 16 años de desarrollo y más de 8476 pacientes asistidos, sigue estos lineamientos, al desarrollar un sistema integral de asistencia continua, que le ha valido el premio de la Academia Nacional de Medicina ${ }^{(6)}$. En esta dirección se plantea cada situación, trabajando desde la perspectiva humanista de la medicina centrada en el paciente y la familia, y se jerarquiza el padecimiento sobre la enfermedad ${ }^{(7,8)}$.

$\mathrm{Al}$ ingreso de un paciente a un servicio de cuidados paliativos, lo primordial es controlar los problemas más frecuentes que, en general, son los síntomas físicos más urgentes, como dolor, disnea, confusión, depresión con riesgo suicida, entre otros, siguiendo los protocolos establecidos $^{(9)}$. Luego, por orden de importancia, se van manejando el resto de los síntomas físicos, emocionales y el aspecto nutricional. Todo esto se realiza mediante un vínculo profesional y humano, que fomente la autonomía del paciente con el equipo interdisciplinario, y que ponga al paciente en una situación de control de síntomas.

$\mathrm{Al}$ abordar el tema de la nutrición, el objetivo puede estar orientado a mejorar los parámetros nutricionales o a lograr un bienestar subjetivo en el paciente y en la familia. Es fundamental considerar la importancia y la significación afectiva y simbólica que le da el paciente y su entorno a la alimentación. Abordar los aspectos psicológicos del paciente y su familia en la esfera nutricional en cuidados paliativos implica para el equipo tratante:

- La capacitación y el abordaje integral de la nutrición desde la interdisciplinariedad.

- Realizar un diagnóstico situacional.

- Describir el impacto psicológico de la nutrición en las diferentes etapas de la evolución de la enfermedad, y en función de su patología de base.

\section{CAPACITACIÓN Y ABORDAJE INTEGRAL DE LA NUTRICIÓN DESDE LA INTERDISCIPLINARIEDAD EN LOS EQUIPOS DE CUIDADOS PALIATIVOS}

Los pacientes portadores de enfermedades avanzadas tienen una incidencia de malnutrición entre el $50 \%$ y el $75 \%$. De allí parte la importancia de abordar este aspecto en la asistencia del paciente en fases avanzadas de la enfermedad ${ }^{(10,11)}$. Los parámetros nutricionales (sintomáticos o analíticos) son uno de los indicadores más fiables para el pronóstico de la supervivencia en estos enfermos, por esto es fundamental la temática de la nutrición en los pacientes portadores de enfermedades avanzadas ${ }^{(12)}$.

El tratamiento dietético se debe ir adaptando constantemente a la evolución clínica de la enfermedad (terreno, comorbilidades, efectos secundarios de los tratamientos convencionales y no convencionales, entre otros). Para ello se debe instruir tanto al paciente como a la familia para una mejor adaptabilidad de que es lo que se espera en cada etapa. En esta instancia se debe construir una nueva forma de alimentación para el paciente, que se naturalice e incorpore a la vida cotidiana.

Existen diferentes vías de alimentación: oral, parenteral, enteral.

- Vía oral: se va estimulando el apetito, cuidando las cantidades, olores, sabores, preferencias en consistencia, en formas de elaboración, frecuencia de las comidas y contexto en el cual se alimenta, reconociendo que comer es un acto social ${ }^{(13)}$.

- Vía enteral por sonda nasogástrica: pacientes con este tipo de sonda para alimentación requieren trabajar la adaptación en la manera de alimentarse, respirar y descansar. Cuando se usa como forma de evitar el líquido de retención, a nivel del tracto digestivo alto, es de suma importancia dar información de la función de esta maniobra y de los motivos de la toma de esta decisión. Si el paciente se encuentra en su domicilio con sonda nasogástrica se orienta al familiar en el manejo de esta, indagando los temores y preocupaciones.

- Gastrostomía: implica el cambio de una función y del esquema corporal. Es necesario el abordaje desde la etapa preoperatoria, y trabajar los miedos y las dudas, explicando lo necesario de esta intervención, las finalidades de la misma y su carácter transitorio o definitivo. Luego de realizarse se debe orientar el aprendizaje en la elaboración de los alimentos y la administración de la medicación por la misma, así como la higiene y el cuidado.

- Vía parenteral: habitualmente de uso intrahospitalario en cuidados paliativos, dado que normalmente se realiza por vía venosa central.

Tanto la alimentación enteral como parenteral son indicaciones médicas, y debemos contar con el consentimiento del paciente para realizarlas. En todas estas formas de alimentación se considera de igual importan- 
cia educar al paciente en la degustación de alimentos en la cavidad oral para mantener el placer del gusto, que va más allá de la saciedad, lo que se busca es el confort.

Otra gran área de interés es abordar los cambios impuestos en la alimentación por tratamientos alternativos que decide el paciente o su familia, a veces, sin consultar al equipo tratante. Estos pueden ser dieta cetogénica, dieta adjunta a medicación ayurvédica, ingesta de azúcares, hongos, gorgojos, entre otros. En las terapias alternativas, muchas veces controversiales, pacientes o familiares depositan allí su esperanza de curación y fantasía de revertir la situación. No es raro que se lo oculten al equipo médico. Estas dietas pueden tener consecuencias en la salud física como en la emocional, ya que el paciente se siente defraudado a largo plazo, al no percibir ninguna mejoría, o incluso algunas de estas dietas tienen riesgos para la salud ${ }^{(14,15)}$.

Brindar asistencia paliativa al paciente en su domicilio tiene ventajas, ya que permite conocer su entorno y propicia este tipo de conversaciones, habilita al paciente a comunicar estas medidas adoptadas, y busca en el equipo tratante la aprobación o consejo sobre esta decisión. Esta instancia de apertura permite trabajar fantasías, mitos, expectativas del paciente o familia sobre las medidas tomadas, lo que logra reflexionar sobre la defraudación y la frustración, tratando de adaptarse más a la situación real, sin que esto repercuta tanto a nivel físico como emocional. En la búsqueda de tratamientos alternativos se ve reflejada la esperanza y la necesidad de tener fe en algo que modifique las condiciones que impone la enfermedad ${ }^{(16)}$.

Abordar estos aspectos es importante porque permite profundizar en valores, significado y propósito de la persona, lo que habilita a abordar la dimensión espiritual de los cuidados paliativos, como dimensión imprescindible para brindar atención de calidad ${ }^{(17)}$.

\section{DIAGNÓSTICO DE LA SITUACIÓN POR EL EQUIPO ASISTENCIAL}

Inicialmente es necesario el abordaje de las expectativas del paciente y familia respecto a la nutrición. De acuerdo con el planteamiento del paciente y su situación. Se fijan objetivos realistas, orientados a adecuar las expectativas y contemplar la frustración cuando estas no se cumplan. La alimentación artificial (enteral y parenteral) es un tratamiento médico en sí mismo y no simplemente un cuidado de base.

Mediante la escucha empática y compasiva del equipo tratante, el paciente se siente habilitado a plan- tear sus expectativas, miedos, temores, objetivos de los tratamientos y dudas ante la enfermedad. En este proceso se van identificando los recursos personales del paciente y la familia. Uno de los objetivos del equipo asistencial es llegar a un acuerdo entre las expectativas del paciente y su familia, y lo que se puede ofrecer para intentar cumplirlas. Es fundamental dar al paciente un papel activo en la toma de decisiones desde la perspectiva bioética ${ }^{(18)}$.

El paciente y su familia deben conocer el valor que tiene la alimentación en las distintas etapas de su enfermedad: su importancia cuando realizan tratamientos específicos de quimio o radioterapia, y cuando se encuentra en la etapa de tratamiento sintomático exclusivo.

En etapas avanzadas se debe buscar la mejor manera de informar, en forma paulatina y cuidadosa, al paciente y su familia que la desnutrición es irreversible, y es parte del proceso en estadios avanzados de enfermedad, y que intentar revertir la situación es una medida fútil que va a generar más complicaciones que beneficios. La información adecuada y tolerable por el paciente evita fomentar falsas expectativas y aceptar esta etapa de su enfermedad.

Si bien el paciente y la familia traen a cada consulta o entrevista, como principal demanda, la nutrición, muchas veces el equipo tratante no la identifica como prioridad, y no jerarquiza el papel que tiene en el padecimiento del paciente y la familia. La alimentación, como necesidad básica del ser humano, se ve alterada, y es fuente universal de sufrimiento tanto para el paciente como para la familia, por lo cual es una preocupación reiterada en la clínica ${ }^{(19)}$.

Esta demanda, que es fuente de sufrimiento, se debe abarcar por todo el equipo asistencial (médicos, enfermeros, psicólogos, fisiatra, terapista ocupacional). En lo que refiere propiamente a los aspectos psicológicos, se busca abordar la ansiedad y la angustia que genera la falta de apetito, y la impotencia que tiene el querer comer y no poder. La complejidad se da ante el deseo de comer y no poder debido a la enfermedad. Este hecho confronta al paciente entre lo que quiere y lo que el cuerpo le permite. El equipo profesional ayuda en esta negociación (cuerpo-mente), donde el paciente debe tener tolerancia ante la falta de apetito, y aceptarla. Además, tiene el desafío de encontrar una nueva forma de alimentarse, que busca estimular de otra forma el deseo de comer. Asimismo, se debe abordar en forma concomitante la frustración de la familia o cuidador cuando no se cumple con el objetivo de alimentar al paciente ${ }^{(20-22)}$. 


\section{IMPACTO PSICOLÓGICO DE LA NUTRICIÓN EN LAS DIFERENTES ETAPAS DE LA EVOLUCIÓN DE LA ENFERMEDAD Y EN FUNCIÓN DE LA PATOLOGÍA DE BASE}

Es importante diferenciar la evolución nutricional de pacientes portadores de enfermedades oncológicas y no oncológicas. En los pacientes con cáncer, la repercusión nutricional puede llegar en algunos casos a ser más severa, la cual llega con frecuencia a la caquexia refractaria, lo que genera alto impacto en el paciente y su entorno.

En primera instancia, el paciente tiene su capacidad funcional relativamente conservada, con Eastern Cooperative Oncology Group - Performance Status (ECOG) 1 o 2, y acude solo o acompañado a la consulta (habitualmente en consulta externa), donde manifiesta como preocupación un cambio en las necesidades básicas de sueño, alimentación y actividades rutinarias.

En este contexto, las primeras manifestaciones clínicas de una enfermedad que subyace en la esfera nutricional pueden ser anorexia, repugnancia selectiva, adelgazamiento, alteraciones del gusto. Esto genera en el paciente y la familia angustia, ansiedad, demandas de resolución del síntoma, buscando en la asistencia una rápida respuesta. Ante esta demanda, el equipo debe estar atento al aspecto subjetivo nutricional, tanto como el objetivo, y reconocer que el efecto de comer traerá una mejoría anímica, que podrá repercutir globalmente en el bienestar y en la calidad de vida. En segunda instancia, cuando el paciente va perdiendo la capacidad funcional ECOG 3 o 4, y el apetito o el deseo de alimentarse disminuye, debemos orientar al familiar $y$ al paciente en cuanto a la forma y los objetivos de la alimentación. El paciente debe tener la libertad de elegir su alimentación, y el equipo trabajará para evitar la culpa por no poder comer lo que su familia exige ${ }^{(23)}$.

Es fundamental empatizar con el paciente cuando no tiene apetito y no obligarlo a la ingesta de alimentos. En forma paralela, debemos manejar la ansiedad y la frustración de los cuidadores cuando el paciente no siente deseos de comer. Es importante trabajar sobre las expectativas de familiares y cuidadores acerca del impacto de la nutrición en el estado general del paciente, y desmitificar la creencia de que la alimentación generará un cambio significativo en la condición del paciente.

La familia aceptará siempre mejor que su ser querido tenga alimentación de confort que ninguna alimentación, aunque las cantidades de este tipo de alimentación sean mínimas. En la tercera instancia, donde el paciente empieza a deteriorar la conciencia (situación de últimos días), se debe trabajar en la fantasía de la ganancia del comer y tomar, sabiendo que en esta etapa no se siente hambre. Este aspecto, si se venía elaborando anteriormente, se maneja en forma adecuada por el familiar. En esta etapa se orienta al familiar sobre qué es lo esperable, se educa sobre medidas de confort y cuidado. Se le explica que el organismo no requiere alimentación alguna y qué el hacerlo podría perjudicar al paciente.

Los estudios realizados demuestran claramente que los enfermos en los últimos días de vida no experimentan hambre, aunque no ingieran ningún alimento. Se debe trabajar en el sufrimiento del familiar-cuidador, porque aquí es donde habitualmente se materializa la idea del que el paciente va a fallecer ${ }^{(24)}$. Durante el duelo de los familiares, el hecho de haber brindado alimento y confort al ser querido va a generar en el familiar/cuidador tranquilidad y una sensación reconfortante ${ }^{(25)}$.

\section{CONCLUSIONES}

El trabajo en equipo, basado en un vínculo de continuidad en todos los ámbitos asistenciales, genera una comunicación fluida, que permite entender la perspectiva del paciente, y dar la importancia integral a la nutrición en la enfermedad avanzada y progresiva. La anorexia, la desnutrición y la pérdida de la imagen corporal genera un impacto psicológico en el paciente, la familia y el equipo. Abordar el impacto en las diferentes etapas de la enfermedad es fundamental a la hora de dar una asistencia de calidad.

Se impone la capacitación de todo el equipo asistencial en la formación en el cuidado nutricional de los pacientes y el acompañamiento psicológico, desde la interdisciplina, haciendo un abordaje específico de las diferentes etapas tanto del paciente como de la familia en cuidados paliativos.

\section{Financiación}

Ninguna.

\section{Conflictos de interés}

Los autores declaran no tener conflictos de intereses.

\section{Declaración de autoría}

LR, VL, GPA contribuyeron igualmente para la concepción del artículo. LR y VG redactaron el artículo. Todos los autores revisaron el artículo y validaron su versión final. 


\section{Referencias bibliográficas}

1. Gómez-Batiste X, Stjernsward J, Espinosa J, MartínezMuñoz M, Trelis J, Constante C. How to design and implement palliative care public health programmes: foundation measures. An operational paper by the WHO Collaborating Centre for Public Health Palliative Care Programmes at the Catalan Institute of Oncology. BMJ Support Palliat Care. 2013;3(1):18-25. doi: 10.1136/bmjspcare-2012-000215.

2. Ferris FD, Gómez-Batiste $\mathrm{X}$, Fürst $\mathrm{CJ}$, Connor $\mathrm{S}$. Implementing quality palliative care. J Pain Symptom Manage. 2007;33(5):533-41. doi: 10.1016/j.jpainsymman.2007.02.033.

3. Piedrafita-Susín AB, Yoldi-Arzoz E, Sánchez-Fernández M, Zuazua-Ros E, Vázquez-Calatayud M. Percepciones, experiencias y conocimientos de las enfermeras sobre cuidados paliativos en las unidades de cuidados intensivos. Enferm Intensiva. 2015;26(4):153-65. doi: 10.1016/j.enfi.2015.06.001.

4. Bruera E, Yennurajalingam S. Palliative care in advanced cancer patients: how and when? Oncologist. 2012;17(2):267-73. doi: 10.1634/theoncologist.2011-0219.

5. Gómez-Batiste X, Ferris F, Paz S, Espinosa J, Porta-Sales J, Stjernswärd J, et al. Ensure quality public health programmes: A Spanish model. Eur J Palliat Care. 2008;15(4):194-9.

6. Modelo asistencial del paciente en etapa terminal de la enfermedad. Resultado de un sistema integral [Internet]. Academia Nacional de Medicina (Consultado el 1 de diciembre de 2020). Disponible en: https://www.anm.org.uy/index_htm files/ganadores\%20gran\%20premio\%20anm.pdf

7. Laura RC. Humanization: a conceptual and attitudinal problem. Physiol Behav. 2017;176(5):139-48.

8. March JC. Humanizar la sanidad para mejorar la calidad de sus servicios. Rev Calid Asist. 2017;32(5):245-7. doi: 10.1016/j. cali.2017.10.001.

9. Píriz Álvarez G, Gómez Sancho M, Ojeda Martín M. Cuidados paliativos. Control de síntomas. 7. a edición. Montevideo, Uruguay. 2014.

10. Maltoni M, Caraceni A, Brunelli C, Broeckaert B, Christakis $\mathrm{N}$, Eychmueller S, et al. Prognostic factors in advanced cancer patients: Evidence-based clinical recommendations - A study by the steering committee of the European Association for Palliative Care. J Clin Oncol. 2005;23(25):6240-8. doi: 10.1200/JCO.2005.06.866.

11. O'Gorman P, McMillan DC, McArdle CS. Impact of weight loss, appetite, and the inflammatory response on quality of life in gastrointestinal cancer patients. Nutr Cancer. 1998;32(2):76-80. doi: 10.1080/01635589809514722.

12. Hui D, Dev R, Bruera E. The last days of life: Symptom burden and impact on nutrition and hydration in cancer patients. Curr Opin Support Palliat Care. 2015;9(4):346-54. doi: 10.1097/ SPC.0000000000000171.
13. Gómez Sancho M, Altisent Trota R, Bátiz Cantera J, Casado Blanco M, Ciprés Casasnovas L, Gándara del Castillo Á, et al. Ética de la alimentación y la hidratación al final de la vida. (Consultado el 1 de octubre 2020). Disponible en: http:// infocuidadospaliativos.com/wp-content/uploads/2016/10/ etica_alimentacioo\%CC\% $81 n$ _fin_de_la_vida_octubre16.pdf

14. Weber DD, Aminzadeh-Gohari S, Tulipan J, Catalano L, Feichtinger RG, Kofler B. Ketogenic diet in the treatment of cancer - Where do we stand? Mol Metab. 2020;33:102-21. doi: 10.1016/j.molmet.2019.06.026.

15. Arends J, Bachmann P, Baracos V. ESPEN guidelines on nutrition in cancer patients. Clin Nutr. 2017;36(1):11-48. doi: 10.1016/j.clnu.2016.07.015.

16. Hopkinson J, Wright D, Corner J. Exploring the experience of weight loss in people with advanced cancer. J Adv Nurs. 2006;54(3):304-12. doi: 10.1111/j.1365-2648.2006.03818.x.

17. Cooper C, Burden ST, Cheng H, Molassiotis A. Understanding and managing cancer-related weight loss and anorexia: Insights from a systematic review of qualitative research. J Cachexia Sarcopenia Muscle. 2015;6(1):99-111. doi: $10.1002 /$ jcsm. 12010 .

18. Barbero J, Pados C, González A. Hacia un modelo de toma de decisiones compartidas para el final de vida. Psicooncología. 2011;8:143-68. Doi: 10.5209/rev_PSIC.2011.v8.n1.11.

19. Benarroz O, Faillace GB, Barbosa LA. Bioética e nutrição em cuidados paliativos oncológicos em. Cad Saude Publica. 2009;25(9):1875-82. doi: 10.1590/s0102$311 \times 2009000900002$.

20. Hutton JL, Baracos VE, Wismer W V. Chemosensory dysfunction is a primary factor in the evolution of declining nutritional status and quality of life in patients with advanced cancer. J Pain Symptom Manage. 2007;33(2):156-65. doi: 10.1016/j.jpainsymman.2006.07.017.

21. Pérez-Cruz PE, Acevedo F. Escalas de estado funcional (o performance status) en cáncer. Gastroenterol Latinoam. 2014;25:219-26. doi: 10.0716/gastrolat2014n300007.

22. Plonk WM, Arnold RM. Terminal care: The last weeks of life. J Palliat Med. 2005;8(5):1042-54. doi: 10.1089/ jpm.2005.8.1042.

23. Fascioli A. Los cuidados paliativos al final de la vida: expresión del reconocimiento del otro. Enfermería Cuid Humaniz. 2016;5(2):46.

24. Loofs TS, Haubrick K. End-of-life nutrition considerations: Attitudes, beliefs, and outcomes. Am J Hosp Palliat Care. 2020:1049909120960124. doi: $10.1177 / 1049909120960124$.

25. Benito E, Dones M, Babero J. El acompañamiento espiritual en cuidados paliativos. Psicooncologia. 2016;13(2-3):367-84. doi: 10.5209/PSIC.54442. 